\title{
In silico Anticancer activity and Caspase targeted study of Saponin rich fraction extracted from Caralluma fimbriata (Wall).
}

\author{
Research Article
}

\section{Desai Tanvi1 $^{*}$, Joshi Shrikant²}

1. Assistant Professor, Department of Pharmacology, Shree Dhanvantary Pharmacy College, Kim, Gujarat. 2. Associate Professor, Department of Pharmacology, Maliba Pharmacy College, Bardoli, Gujarat.

\begin{abstract}
Caralluma fimbriata (Wall.) (Asclepiadaceae), is mentioned as vegetable in Indian Materia Medica and an affluent resource of saponins. It is reported in conventional medicine method of India as well as Arabia that $C$. fimbriata was extensively used for cancer treatment. Current study was planned to assess anticancer potential of saponin rich fraction from $C$. fimbriata using in silico and in vitro assays. Caratubersides A-G, a pregnane glycosides found in C. fimbriata were taken for in silico examination and processed through PASS Online software for the prediction of structure dependent pharmacological actions. Docking was carried out using Autodock Tool and Autodock Vina, revealed antineoplastic action of caratubersides along with apoptogenic potential. MTT assay was performed on MCF-7 cell line. Shell less chicken embryo culture assay was done for anti-angiogenic properties at different concentrations $(1.5 \mu \mathrm{g} / \mathrm{ml}, 3 \mu \mathrm{g} / \mathrm{ml}$, and $6 \mu \mathrm{g} / \mathrm{ml})$. Chromosomal aberrations assay was carried out in cultured human blood. And apoptogenic potential was estimated on MCF-7 cells using cleaved caspases 3 and caspase 8 cell based ELISA assay kit. Results of study showed that IC50 of saponin rich fraction of C. fimbriata was at $3 \mu \mathrm{g} / \mathrm{mL}$. Considerable $(\mathrm{p}<0.05)$ decreases were observed in angiogenic properties. Insignificant chromosomal aberrations were found in normal cells. Treatment of saponin rich part improved levels of caspases 3 as well as caspase 8 (ODs 1.35 and 1.68 respectively). From the study, saponin rich portion obtained from C. fimbriata displayed antiproliferative, anti-angiogenic actions along with apoptogenic prospective and no significant chromosomal aberrations were found in normal human cells.
\end{abstract}

Key Words: Caralluma fimbriata, In silico, MTT assay, Angiogenesis, Chromosomal Aberrations assay, Apoptosis.

\section{Introduction}

Upsurge in numbers of deaths related to cancer along with unfavourable and deadly side effects of cancer treatments like radiation and chemotherapy, the interest in searching for novel cancer treating agents, especially of plants origin, is on rise (1). C. fimbriata has synonym Caralluma ascendens is a widespread, juicy cactus as well as feral remedial plant belongs to Asclepiadaceae family (2). In Western India it is also called Shindula makadi, Makad shenguli, Kullimudayan, and Ranshabar (3). The plant is widely found in Africa, India, the Canary Islands, Southern Europe and Arabia (4).

C. fimbriata is marked as a hunger suppressant, vegetable and thirst quencher in Indian Materia Medica and grow as an arid herb in waterless places of India (5). C. fimbriata is also used to make boundaries around gardens. Plant is also used to make chutneys and pickles in numerous centuries (6). C. fimbriata is reported as an

* Corresponding Author:

\section{Desai Tanvi}

Assistant Professor,

Department of Pharmacology,

Shree Dhanvantary Pharmacy College,

Kim, Gujarat India.

Email Id: tanvidesai89@yahoo.in antiobesogenoic agent and metabolic regulator (7). It is considered to be "famine food" which repress hunger and satisfy thirst. It is a "portable food" which prevents starvation in period of frantic requirement of food while travelling (8). C. fimbriata is safe and utilized conventionally for treating paralysis, rheumatic disorders, inflammation and diabetes along with leprosy. It showed antimalarial, antiproliferative, antiulcer, antinociceptive, antitrypanosomal, antioxidant activities, antihyperglycaemic, hypolipidaemic and hepatoprotective activity $(9,10)$. Devi and her friends in 2016 reported that the plant is extensively useful in the Indian traditional medication system for anticancer activity (11). Qayyum in 2018 reported that traditionally Caralluma species used for cancer treatment (12). Zari and his colleagues 2018 reported that in the Indian and Arabic traditional medicine Caralluma species are used in the cancer therapy (13). Earlier Shenai et al., observed that ethanolic extract of $C$. fimbriata leaves having cytotoxicity in COLO 320 cells (1). Also AlFaifi investigated in vitro antiproliferative action of Caralluma spp. in HEPG2 and MCF-7 cell lines (14).

C. fimbriata is a rich source of pregnane glycosides, megastigmane glycosides, steroidal glycosides, saponins, flavonoids and flavone glycosides (15), pregnane steroids, hexadecanoic acid, oleic acid, aromatic and nonaromatic bitter principles, alkaloids, unsaturated and saturated hydrocarbons, volatile compounds along with $\beta$-sitosterol. There are also few 
active elements present in C.fimbriata like Caratubersides (16), Boucerosides and Tomenkogenin (17).

Saponins are obtains as secondary metabolites from plants and they are glycosides in nature which exerts various pharmacological effects. Previous studies demonstrated anti-proliferative, anti-angiogenic, and antimetastatic activity of saponins through induction of apoptosis and cell differentiation. Saponins also showed reversal of multidrug resistance in some cancers (18). Moreover, steroidal class of saponins, Pregnan glycosides, was reported to have potent anticancer and pro-apoptotic activity. Caratubersides available in $C$. fimbriata are pregnane glycosides which were ill reported for their cytotoxic or apoptogenic potential. This promoted us to explore anticancer effects of saponin rich extract of $C$. fimbriata using in vitro models (19).

Here, structural dependent biological activities of caratubersides (A to $G$ ) were predicted using PASS Online and Swiss Target Prediction Software and structural docking was done via Autodock Tools and Autodock Vina. Additional saponin rich fraction was isolated from $C$. fimbriata leaves using water and nbutanol fractionation and studied further for anticancer actions. Cell viability and cell toxicity (MTT) assay was performed to evaluate antiproliferative activity on MCF-7 cells. Shell less chicken embryo culture assay was performed to check anti-angiogenic property. Effects on physical reliability of genetic materials were studied using cultured human blood in Chromosomal aberrations assay. Apoptogenic prospective was estimated on MCF-7 cells through cleaved caspase 3 as well as caspase 8 cell based ELISA assay kit.

\section{Materials and Methods Materials}

Breast cancer MCF-7 cell line was attained from NCCS, Pune, Maharashtra.

Dried hydroalcoholic $C$. fimbriata leaves extract was procured from Navchetna kendra, New Delhi, India.

Prolific eggs obtained through egg seller. And ELISA assay kit was obtained from Cell Signaling Technology.

\section{Isolation of Saponin from Caralluma fimbriata}

In hydroalcoholic extract of $C$. fimbriata leaves (5gm), $50 \mathrm{ml}$ distilled water was added and shifted the mixture in separating funnel. The same amount of nbutanol $(10: 10 \mathrm{v} / \mathrm{v})$ was placed in separating funnel and permitted them for overnight separation of n-butanol fraction at room temperature. At $45^{\circ} \mathrm{C}$ collected nbutanol part was evaporated to get saponin rich extract (20).

\section{Fingerprinting of Caralluma fimbriata}

Fingerprinting study of $C$. fimbriata was performed by the use of CAMAG HPTLC equipment prepared by Linomat V applicator, TLC scanner 3, Reprostar 3 as well as 12 bit CCD camera for documents of photos and restricted with software
WinCATS-4. The samples $(30 \mu \mathrm{L})$ were placed in band form which containing $6 \mathrm{~mm}$ width by the use of Camag microlitre plunger on $200 \mu \mathrm{m}$ thick silica gel coated aluminum plate $(5 \times 10 \mathrm{~cm})$. Leaves extract as well as $\beta$-sitosterol standard were solubilized in small quantity of methanol. Samples were prepared in concentration of $10 \mu \mathrm{g} / 10 \mu \mathrm{l}$. The plate was developed till $85 \mathrm{~mm}$ distance in a glass chamber $30 \mathrm{~min}$ formerly saturated with toluene: ethyl acetate: methanol: glacial acetic acid (8:1:0.5:0.3v/v) solvent system. Air dried the plat for removal of mobile phase and then derivatized the plat by dripping in anisaldehyde- $\mathrm{H} 2 \mathrm{SO} 4$ reagent till 2 seconds. After that dried the plate in air and heated it till 10 minutes at $110^{\circ} \mathrm{C}$. Then scanning was executed at $\lambda=584 \mathrm{~nm}$ with Camag TLC scanner (21).

\section{In silico studies \\ Prediction of structure base biological activities in PASS Online}

For caratubersides $A$ to $G$, structures of them were draw in ChemDraw Ultra 10.0 and then save them in .cdx file formates which were then opened in Chem3D Ultra 10.0 to converted them in .mol file extension. These MDL Mol file were then opened in PASS online software for prediction of activities. For caspase 3 as well as for caspase 8 stimulation resultant values $\mathrm{Pa}$ and $\mathrm{Pi}$ (probability to be active and probability to be inactive respectively) were search and adopted from different predictable pharmacological actions $(22,23,24)$.

\section{Molecular docking}

For docking studies .mol files of caratubersides (A-G) were changed to .pdb format (PDB) by OpenBabel 2.4.1software. Caspase 3 (3DEI) as well as caspase 8 (3KJQ) configurations was acquired in .pdb file format through RCSB data bank. After that they were opened through AutoDock Tools 4.2.6 and processed for obtaining .pdbqt files. MetaPocket 2.0 was used to recognize different binding sites for ligands on caspase 3 and caspase 8 and to choose parameters for grid box. Values of grid box were utilized for docking of related proteins and caratubersides in Autodock Vina $(25,26)$.

\section{MTT assay}

MTT assay was carried out for evaluation of antiproliferative activity of saponin rich part from C.fimbriata in MCF-7 cells line with different $(0.001 \mu \mathrm{g} /$ $\mathrm{ml}, \quad 0.01 \mu \mathrm{g} / \mathrm{ml}, 0.1 \mu \mathrm{g} / \mathrm{ml}, 1 \mu \mathrm{g} / \mathrm{ml}$ and $10 \mu \mathrm{g} / \mathrm{ml})$ concentrations. Saponin rich fraction of C.fimbriata $1 \mathrm{mg} / \mathrm{ml}$ was solubilized in to DMSO, then consecutively diluted by growth media for the different. Concentration of DMSO was reserved less than $0.1 \%$. MCF-7 cell line was maintained within DMEM having $10 \%(\mathrm{v} / \mathrm{v})$ bovine serum from fatal calves, were placed into 96 well plates. Treatments of different concentrations of saponin wealthy portion was given to cells and place the plate in incubator at $37^{\circ} \mathrm{C}$ and $5 \%$ CO2 till 4 days. After 96 hours cell were treated with MTT dye and again incubated till 4 hours. Cells were formed purple colour formazan products and then 
DMSO was added in wells. DMSO dissolved the formazan products which were measured spectrophotometrically on $550 \mathrm{~nm}$ to obtain IC50 value (27).

\section{Shell less chicken embryo culture assay}

Fertilized eggs cooled till 25 minutes and cleaned through $70 \%$ alcohol for reduction of outside effluence after $72 \mathrm{hrs}$ of incubation. Albumin of nonfertile eggs was placed in sterilized Petridish, to provide pad for egg contains. After that incubated eggs were break from top by using spatula and filling of eggs were smoothly discharge above albumin bed in Petridish. Effectively placed embryos were separated in the control and treatment groups. In treatment groups treatment with various concentrations $(1.5 \mu \mathrm{g} / \mathrm{ml}, 3 \mu \mathrm{g} /$ $\mathrm{ml}$, and $6 \mu \mathrm{g} / \mathrm{ml}$ ) of saponin loaded part of C. fimbriata was given in the dosage of $10 \mu 1$ on blood vessels developed area. All Petridish were enclosed and again incubated at $37 \pm 0.5^{\circ} \mathrm{C}$ and $80 \%$ humidity. Later than at 3hours and 6 hours, differences in quantity of extremities, junctions, nodes, branches, total branches length were examined via angiogenesis analyzer ImageJ 1.50 b software (28).

\section{Chromosomal aberrations (CA) assay}

Fresh blood samples were collected in four vials from healthy, non-smoking and erratically chosen female and male (2:1) between $20-25$ years of age. One vial was marked as untreated control and remaining three were marked as treated vial. Treatment by three different concentrations $(1.5 \mu \mathrm{g} / \mathrm{ml}, 3 \mu \mathrm{g} / \mathrm{ml}$ and $6 \mu \mathrm{g} / \mathrm{ml})$ was given in a dosage of $50 \mu \mathrm{l}$. In to each vials $5 \mathrm{ml}$ PBMAX TM karyotyping growth medium, 50 $\mu 1$ Heparin and $0.6 \mathrm{ml}$ fresh blood was added and allowed to incubate them till 72 hours. Later at 24 hours, treatment with various concentrations of saponin wealthy portion was given. On 69th hour cultures were treated with $100 \mu$ l colchicine. On the completion of 72 hours, the vials were centrifuged till 8 minutes on $1200 \mathrm{rpm}$ and discarded the supernatants. After that cells were treated with $5 \mathrm{ml}$ of $0.56 \% \mathrm{KCl}$ and centrifuge tubes were permitted to place in incubator for $25 \mathrm{~min}$ at $37^{\circ} \mathrm{C}$ for the swelling of cells. The tubes were centrifuged again on $1200 \mathrm{rpm}$ till $8 \mathrm{~min}$. Supernatants were removed, cells were fixed by addition of $6 \mathrm{ml}$ of carnoy's fixative and placed them into refrigerator till 1 hour. The samples were centrifuged again for $8 \mathrm{~min}$ at $1200 \mathrm{rpm}$, supernatants were discarded and using $3 \mathrm{ml}$ of fixative, the washing step was continued for obtaining white pellet. After that slides were prepared through placing 5-7 drops of white pallet from suitable altitude on priorly cooled clean slides. Then coded them and used for scoring after staining with $2 \%$ giemsa stain. 100 metaphases were scored in each group using compound light microscope at $100 \mathrm{x}$ magnifications for chromosomal aberrations (29).

\section{Caspase assay}

Stimulation of caspase 3 as well as caspase 8 was estimated by using ELISA assay kit in breast cancer MCF-7 cell line. The cells were placed into 96 well plates, treated with $10 \mu \mathrm{g} / \mathrm{ml}$ concentration of saponin rich fraction and allowed them to incubate till 6 hours at $37^{\circ} \mathrm{C}$ and $5 \% \mathrm{CO}$. At the end of 6 hours development media was discarded through wells and washed the cells by PBS. Washed cells were infused with triton X $(0.5 \%)$ till 2 minutes and fixed in methanol. Using PBS cells were again rinsed and treated by cleaved caspase- 3 (Asp175) as well as cleaved caspase- 8 (Asp 391) till couple of hours. Rinsed cells 3 times using PBS and treated with corresponding HRP conjugated secondary antibodies till half an hour. After 30 minute cells were rinsed three times, identify through TMB substrate and calculated optical densities using wavelength $450 \mathrm{~nm}$ by Fluostar (BMG Germany) (30).

\section{Statistical analysis}

Results were uttered as mean \pm S.E.M. for each investigational group. Arithmetical examination was carrying out by IBM SPSS Statistics 22 statistical software. Arithmetic assessment among various treatments and control were executed using ANOVA pursued via turkey's post hoc test. Minimum significant level was recognized on $\mathrm{p}<0.05$.

\section{Results}

\section{Isolation of Saponins}

C. fimbriata is a rich source of saponins. We obtained the extraction yield of $35 \%$ of saponins from hydroalcoholic extract of $C$. fimbriata leaves.

\section{Fingerprinting of Caralluma fimbrita}

Chromatograms attained by $\beta$-sitosterol and $C$. fimbriata extract using solvent system toluene: ethyl acetate: methanol: glacial acetic acid $(8: 1: 0.5: 0.3 \mathrm{v} / \mathrm{v})$ were shown in figure 1 and 2 . The $\mathrm{R}_{\mathrm{f}}$ value at $584 \mathrm{~nm}$ was found to be 0.55 (Fig. 1and 2A, 2B).

\section{Figure 1. 3D HPTLC chromatogram for $\beta$-sitosterol and C. fimbriata extract}

Figure 2. HPTLC chromatograms of
(A) $\beta$-sitosterol
(B) C. fimbriata extract

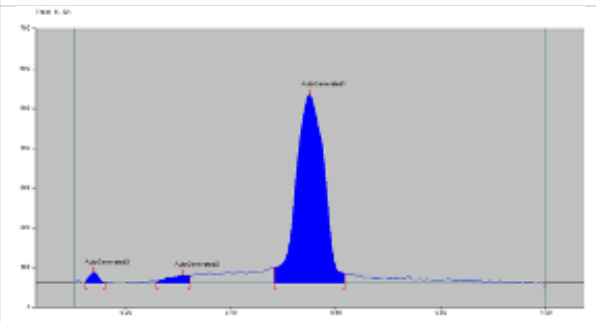

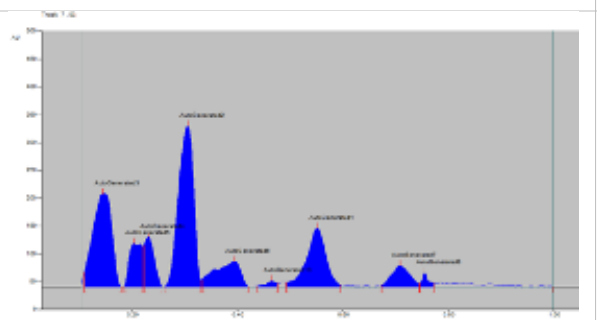


Desai Tanvi et.al., In silico Anticancer activity and Caspase targeted study of Saponins from Caralluma fimbriata (Wall).

In Silico studies

Prediction of biological activities using in silico methods

On the basis of structures biological activities of caratubersides (A-G) were predicted using PASS Online and SwissTargetPrediction Softwares. PASS Online predictions with $\mathrm{Pa}$ values greater than 0.7 were enlisted. The anticancer effect related predictions which were common for all caratubersides were found to include antineoplastic (breast cancer), antiangiogenic activity, apoptosis agonist and caspase 3 as well as caspase 8 stimulation. Also by SwissTargetPrediction apoptotic regulation activities were expected for all caratubersides. For caspase 3 and caspase 8 stimulation values of $\mathrm{Pa}$ along with $\mathrm{Pi}$ were stated separately. (Table 1).

Table 1. Biological activity prediction of caratubersides using in silico methods.

\begin{tabular}{|c|c|c|c|c|c|c|c|c|c|c|c|}
\hline \multirow{3}{*}{$\begin{array}{l}\text { Sr. } \\
\text { No. }\end{array}$} & \multirow{3}{*}{$\begin{array}{r}\text { Triterpenoids / } \\
\text { Structures }\end{array}$} & \multicolumn{5}{|c|}{ Caspases 3 stimulation } & \multicolumn{5}{|c|}{ Caspases 8 stimulation } \\
\hline & & \multicolumn{2}{|c|}{$\begin{array}{l}\text { PASS Online } \\
\text { Prediction }\end{array}$} & \multirow{2}{*}{$\begin{array}{c}\text { Binding } \\
\text { energy } \\
\text { (Kcal/ } \\
\text { mol) }\end{array}$} & \multirow{2}{*}{$\begin{array}{l}\text { Amino } \\
\text { acid } \\
\text { residue }\end{array}$} & \multirow[t]{2}{*}{$\begin{array}{l}\text { Hydrogen } \\
\text { Bond }\end{array}$} & \multicolumn{2}{|c|}{$\begin{array}{l}\text { PASS Online } \\
\text { Prediction }\end{array}$} & \multirow{2}{*}{$\begin{array}{c}\text { Binding } \\
\text { energy } \\
\text { (Kcal/ } \\
\text { mol) }\end{array}$} & \multirow[t]{2}{*}{$\begin{array}{l}\text { Amino } \\
\text { acid } \\
\text { residue }\end{array}$} & \multirow[t]{2}{*}{$\begin{array}{l}\text { Hydrogen } \\
\text { Bond }\end{array}$} \\
\hline & & $\mathrm{Pa}$ & $\mathbf{P i}$ & & & & $\mathbf{P a}$ & $\mathbf{P i}$ & & & \\
\hline 1 & Caratuberside A & 0.857 & 0.004 & -7.9 & $\begin{array}{l}\text { Glu123 } \\
\text { Gly122 } \\
\text { Rxb300 } \\
\text { Gly165 } \\
\text { His121 } \\
\text { Tyr204 }\end{array}$ & 1 & 0.718 & 0.003 & -7.6 & $\begin{array}{l}\text { Thr149 } \\
\text { Pro415 } \\
\text { Tyr412 } \\
\text { Ser316 } \\
\text { His317 }\end{array}$ & 11 \\
\hline 2 & Caratuberside B & 0.773 & 0.007 & -7.9 & $\begin{array}{l}\text { Glu123 } \\
\text { Gly122 } \\
\text { Rxb300 } \\
\text { Gly165 } \\
\text { His121 } \\
\text { Tyr204 }\end{array}$ & 1 & 0.691 & 0.004 & -6.3 & $\begin{array}{l}\text { Thr149 } \\
\text { Pro415 } \\
\text { Tyr412 } \\
\text { Ser316 } \\
\text { His317 }\end{array}$ & 11 \\
\hline 3 & Caratuberside $\mathrm{C}$ & 0.545 & 0.022 & -9.3 & $\begin{array}{l}\text { Glu123 } \\
\text { Gly122 } \\
\text { Rxb300 } \\
\text { Gly165 } \\
\text { His121 } \\
\text { Tyr204 }\end{array}$ & 1 & 0.445 & 0.032 & -8.0 & $\begin{array}{l}\text { Thr149 } \\
\text { Pro415 } \\
\text { Tyr412 } \\
\text { Ser316 } \\
\text { His317 }\end{array}$ & 11 \\
\hline 4 & & 0.495 & 0.028 & -9.4 & $\begin{array}{l}\text { Glu123 } \\
\text { Gly122 } \\
\text { Rxb300 } \\
\text { Gly165 } \\
\text { His121 } \\
\text { Tyr204 }\end{array}$ & 1 & 0.354 & 0.084 & -7.5 & $\begin{array}{l}\text { Thr149 } \\
\text { Pro415 } \\
\text { Tyr412 } \\
\text { Ser316 } \\
\text { His317 }\end{array}$ & 11 \\
\hline 5 & & 0.398 & 0.052 & -9.0 & $\begin{array}{l}\text { Glu123 } \\
\text { Gly122 } \\
\text { Rxb300 } \\
\text { Gly165 } \\
\text { His121 } \\
\text { Tyr204 }\end{array}$ & 1 & 0.354 & 0.084 & -7.9 & $\begin{array}{l}\text { Thr149 } \\
\text { Pro415 } \\
\text { Tyr412 } \\
\text { Ser316 } \\
\text { His317 }\end{array}$ & 11 \\
\hline
\end{tabular}




\begin{tabular}{|c|c|c|c|c|c|c|c|c|c|c|c|}
\hline 6 & Caratuberside $\mathrm{F}$ & 0.470 & 0.032 & -9.1 & $\begin{array}{l}\text { Glu123 } \\
\text { Gly122 } \\
\text { Rxb300 } \\
\text { Gly165 } \\
\text { His121 } \\
\text { Tyr204 }\end{array}$ & 1 & 0.435 & 0.036 & -6.8 & $\begin{array}{l}\text { Thr149 } \\
\text { Pro415 } \\
\text { Tyr412 } \\
\text { Ser316 } \\
\text { His317 }\end{array}$ & 11 \\
\hline 7 & Caratuberside $\mathrm{G}$ & 0.689 & 0.012 & -8.5 & $\begin{array}{l}\text { Glu123 } \\
\text { Gly122 } \\
\text { Rxb300 } \\
\text { Gly165 } \\
\text { His121 } \\
\text { Tyr204 }\end{array}$ & 1 & 0.510 & 0.016 & -7.4 & $\begin{array}{l}\text { Thr149 } \\
\text { Pro415 } \\
\text { Tyr412 } \\
\text { Ser316 } \\
\text { His317 }\end{array}$ & 11 \\
\hline
\end{tabular}

\section{MTT assay:}

Antiproliferative MTT assay for saponin rich part from C.fimbriata was performed on MCF-7 cells line displayed IC50 at $3 \mu \mathrm{g} / \mathrm{ml}$ (Table 2).

Table 2: MTT assay for saponin rich fraction of $C$.fimbriata.

\begin{tabular}{|c|c|}
\hline Concentration & C. fimbriata \\
\hline 10 & 68.75 \\
\hline 1 & 32.41 \\
\hline 0.1 & 22.18 \\
\hline 0.01 & 2.36 \\
\hline 0.001 & 1.28 \\
\hline
\end{tabular}

\section{Shell less chicken embryo culture assay}

There is a considerable $(p<0.05)$ decreases in quantity of angiogenic properties after $0-3$ hour and $0-6$ hour of exposure to different concentration of saponin loaded portion (Table 3 and Fig. 3 ).

Figure 3. Photos of shell less chicken embryo cultures

\section{For control}



0 hour

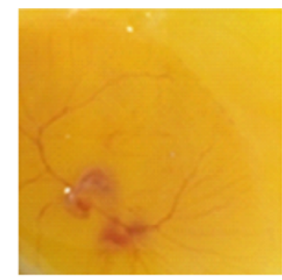

3 hours

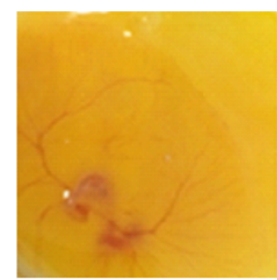

6 hours
For $1.5 \mu \mathrm{g} / \mathrm{ml}$ saponin rich fraction of $C$. fimbriata

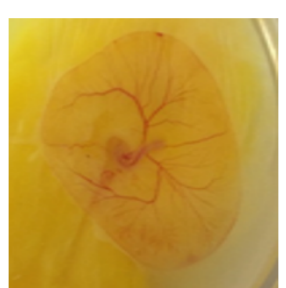

0 hour

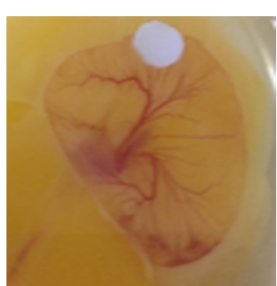

3 hours

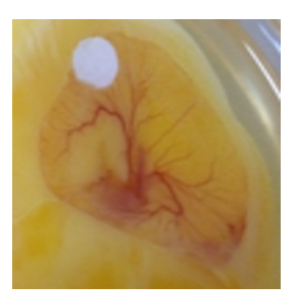

6 hours
For $3 \mu \mathrm{g} / \mathrm{ml}$ saponin rich fraction of $C$. fimbriata

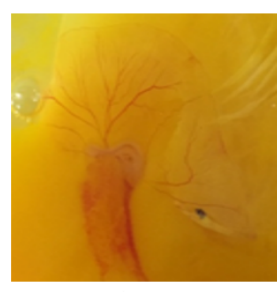

0 hour



3 hours

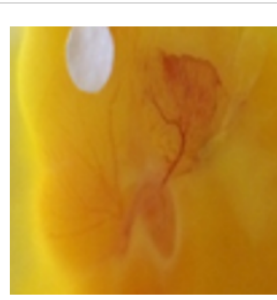

6 hours

For $6 \mu \mathrm{g} / \mathrm{ml}$ saponin rich fraction of $C$. fimbriata

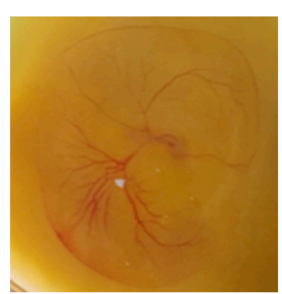

0 hour



3 hours

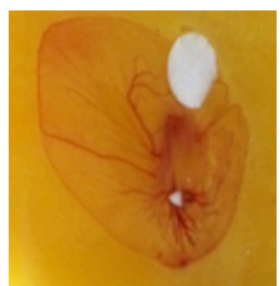

6 hours 
Desai Tanvi et.al., In silico Anticancer activity and Caspase targeted study of Saponins from Caralluma fimbriata (Wall)

Table 3. Effect of saponin rich fraction of $\boldsymbol{C}$. fimbriata on Angiogenic parameters in Shell less chicken embryo culture.

Concentra $\%$ reduction in number \% reduction in number \% reduction in number \% reduction in number \% reduction in total \begin{tabular}{|l|l|l|l|l|l|l} 
tions & of extremities & of nodes & of junction & of branches & branch length
\end{tabular}

\begin{tabular}{|c|c|c|c|c|c|c|c|c|c|c|}
\hline & 0 - 3 hours & 0 -6 hours & -3 hours & 0 -6 hours & 0 -3 hours & 0 -6 hours & 0 -3 hours & 0 -6 hours & 0 -3 hours & 0 -6 hou \\
\hline ontrol & 0 & 0 & 0 & 0 & 0 & 0 & 0 & 0 & 0 & 0 \\
\hline$\mu \mathrm{g} / \mathrm{ml}$ & $\begin{array}{c}26.85 \pm \\
0.99^{*}\end{array}$ & $\begin{array}{c}41.96 \pm \\
0.93^{*}\end{array}$ & $\begin{array}{c}28.75 \pm \\
0.83^{*}\end{array}$ & $\begin{array}{c}48.20 \pm \\
1.07^{*}\end{array}$ & $\begin{array}{c}34.04 \pm \\
1.32 *\end{array}$ & $\begin{array}{c}52.62 \pm \\
1.87^{*}\end{array}$ & $\begin{array}{c}37.74 \pm \\
1.47^{*}\end{array}$ & $\begin{array}{c}55.73 \pm \\
0.95^{*}\end{array}$ & $\begin{array}{c}28.81 \pm \\
0.82 *\end{array}$ & $\begin{array}{c}44.61 \pm \\
1.23^{*}\end{array}$ \\
\hline & $\begin{array}{c}36.57 \pm \\
1.08^{*}\end{array}$ & $\begin{array}{c}60.37 \pm \\
1.32 *\end{array}$ & $\begin{array}{c}35.97 \pm \\
0.95^{*}\end{array}$ & $\begin{array}{c}60.68 \pm \\
1.52 *\end{array}$ & $\begin{array}{c}40.19 \pm \\
0.74^{*}\end{array}$ & $\begin{array}{c}59.02 \pm \\
0.84^{*}\end{array}$ & $\begin{array}{c}45.83 \pm \\
0.83^{*}\end{array}$ & $\begin{array}{c}61.00 \pm \\
1.34^{*}\end{array}$ & $\begin{array}{c}37.08 \pm \\
0.72 *\end{array}$ & $\begin{array}{c}59.88 \pm \\
1.37^{*}\end{array}$ \\
\hline G/II & $\begin{array}{c}42.93 \pm \\
1.04^{*}\end{array}$ & $\begin{array}{c}67.12 \pm \\
1.01^{*}\end{array}$ & $\begin{array}{c}46.57 \pm \\
1.01^{*}\end{array}$ & $\begin{array}{c}67.38 \pm \\
1.64^{*}\end{array}$ & $\begin{array}{c}50.25 \pm \\
1.37^{*}\end{array}$ & $\begin{array}{c}68.04 \pm \\
2.03^{*}\end{array}$ & $\begin{array}{c}51.12 \pm \\
1.25^{*}\end{array}$ & $\begin{array}{c}69.00 \pm \\
0.75^{*}\end{array}$ & $\begin{array}{c}51.54 \pm \\
1.00^{*}\end{array}$ & $\begin{array}{c}68.09 \pm \\
2.45^{*}\end{array}$ \\
\hline
\end{tabular}

Each values stated as mean \pm S.E.M. $(n=6)$ per group. Statistical analysis was performed using one-way ANOVA pursued via Turkey's post hoc test. $* \mathrm{P}<0.05$ contrasts to control.

\section{Chromosomal aberrations (CA) assay:}

$50 \mu \mathrm{l}$ of various concentrations $(1.5 \mu \mathrm{g} / \mathrm{ml}, 3 \mu \mathrm{g} / \mathrm{ml}, 6 \mu \mathrm{g} / \mathrm{ml})$ of saponin wealthy part was placed in cultured vials showed insignificant change in amount of entire chromosomal aberrations contrast to control group (Table 4 and Fig 4).

Table 4. Chromosomal aberrations after treatment

\begin{tabular}{|c|c|c|c|c|}
\hline Aberration & $\begin{array}{l}\text { Normal } \\
\text { (Control) }\end{array}$ & $\begin{array}{l}\text { Saponin rich fraction of } \\
\text { C. fimbriata } \\
(1.5 \mu \mathrm{g} / \mathrm{ml})\end{array}$ & $\begin{array}{c}\text { Saponin rich fraction of } \\
\text { C. fimbriata } \\
(3 \mu \mathrm{g} / \mathrm{ml})\end{array}$ & $\begin{array}{l}\text { Saponin rich fraction of } \\
\text { C. fimbriata } \\
(6 \mu \mathrm{g} / \mathrm{ml})\end{array}$ \\
\hline Chromatid Break & $5.00 \pm 0.57$ & $4.66 \pm 1.76$ & $6.33 \pm 0.88$ & $5.66 \pm 0.88$ \\
\hline Chromosome Break & $3.66 \pm 0.66$ & $3.33 \pm 0.88$ & $5.00 \pm 0.57$ & $6.00 \pm 1.15$ \\
\hline Chromosome Gap & $5.66 \pm 0.88$ & $6.33 \pm 0.88$ & $7.00 \pm 0.57$ & $4.33 \pm 0.33$ \\
\hline Dicentric Fragments & $1.00 \pm 0.57$ & $2.33 \pm 0.33$ & $3.33 \pm 1.45$ & $1.00 \pm 0.57$ \\
\hline Acentric Fragment & $3.33 \pm 0.88$ & $2.33 \pm 0.88$ & $4.33 \pm 1.22$ & $4.66 \pm 1.20$ \\
\hline Ring & $0.33 \pm 0.33$ & $0.00 \pm 0.00$ & $0.00 \pm 0.00$ & $0.33 \pm 0.33$ \\
\hline Premature Separation & $1.00 \pm 1.00$ & $2.00 \pm 1.15$ & $1.66 \pm 0.88$ & $4.00 \pm 2.08$ \\
\hline Endoreduplication & $0.66 \pm 0.66$ & $0.33 \pm 0.33$ & $0.00 \pm 0.00$ & $0.00 \pm 0.00$ \\
\hline Hypodiploid & $8.66 \pm 0.88$ & $9.00 \pm 1.52$ & $11.66 \pm 0.66$ & $13.33 \pm 0.33$ \\
\hline Hyperdiploid & $2.00 \pm 0.57$ & $2.33 \pm 0.88$ & $1.33 \pm 0.33$ & $0.66 \pm 0.66$ \\
\hline
\end{tabular}

Each values stated as mean \pm S.E.M. $(\mathrm{n}=6)$ per group. Statistical analysis was performed using one-way ANOVA pursued via Turkey's post hoc test. $* \mathrm{P}<0.05$ contrasts to control.

Figure 4. Representative pictures of Chromosomal Aberrations

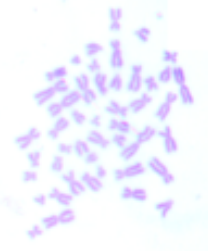

A

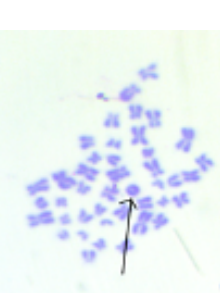

G

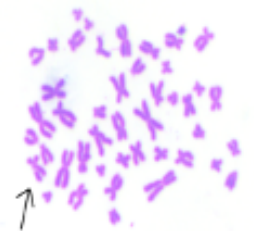

B



$\mathrm{H}$

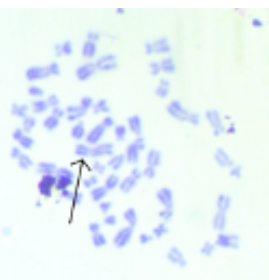

$\mathrm{C}$

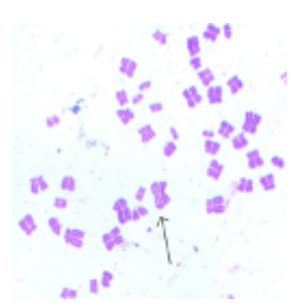

I

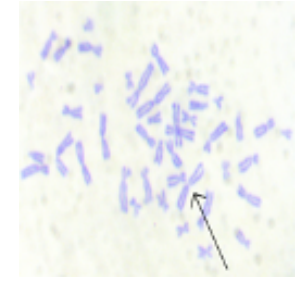

$\mathrm{D}$

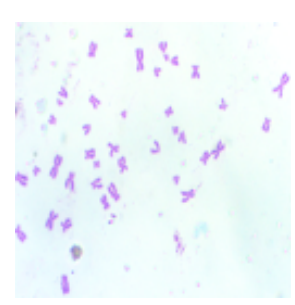

$\mathrm{J}$

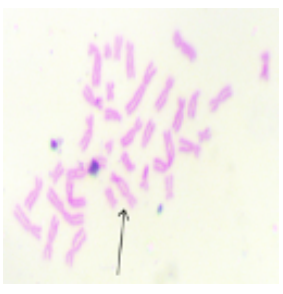

$\mathrm{E}$

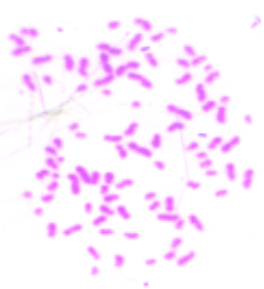

K



F

A: Normal metaphase: Number of chromosomes is from 44 to 48, B: Chromatid break: Break in one sister-chromatid at any one locus, C: Chromosomal break: Breaks in both sister-chromatids at any one locus, D: Chromosomal gap: Gap between the chromosomes from centromere, E: Dicentric fragment: Abnormal chromosome with two centromeres. It is formed through the fusion of two chromosome segments, F: Acentric fragment: A segment of a chromosome that lacks a centromere, G: Ring: A portion of a chromosome has broken off and formed a circle or ring, H: Premature separation: Premature loss of cohesion between centromeres results in their independent segregation at meiosis I, I: Endoreduplication: Replication of the nuclear genome in the absence of mitosis, which leads to elevated nuclear gene content and polyploidy, J: Hypodiploidy: Number of chromosomes are <44, K: Hyperdiploidy: Number of chromosomes are $>48$. 


\section{Caspase assay}

Caspases 3 as well as 8 activations was observed in MCF-7 cancer cell line after treatment. Table 5 and Figure 5 represent the data of stimulation of caspases 3 and 8 (Table 5 and Fig 5).

\section{Table 5. Apoptosis assay of saponin rich fraction of} C. fimbriata for caspases 3 and 8

\begin{tabular}{|c|c|c|c|} 
Treatments & $\begin{array}{c}\text { Concent } \\
\text { rations }\end{array}$ & $\begin{array}{c}\text { OD450 } \\
\text { Caspase 3 }\end{array}$ & Caspase 8 \\
\hline $\begin{array}{c}\text { Saponin rich } \\
\text { part of }\end{array}$ & $10 \mu \mathrm{g} / \mathrm{ml}$ & 1.35 & 1.68 \\
$\begin{array}{c}\text { C. fimbriata } \\
\text { Staurosporine }\end{array}$ & $1 \mu \mathrm{g} / \mathrm{ml}$ & 2.47 & 2.6 \\
\hline
\end{tabular}

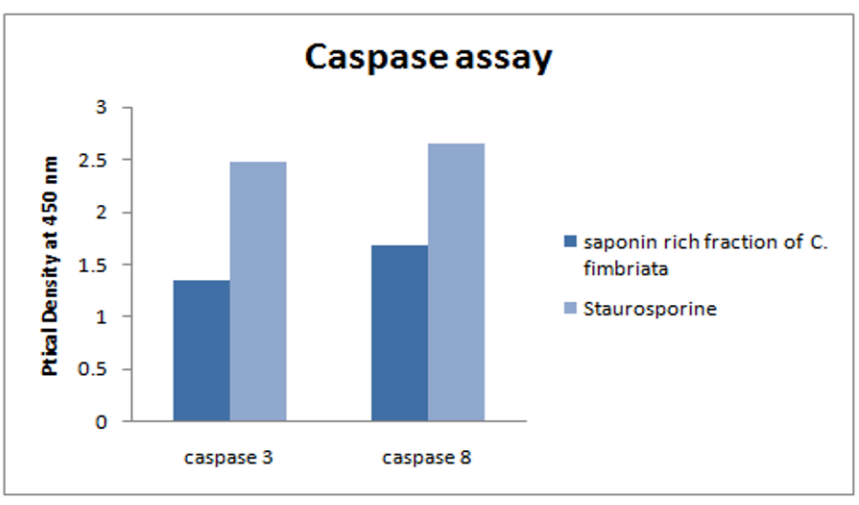

Figure 5. The ODs at $450 \mathrm{~nm}$ in consequent of cleaved Caspase 3 (Asp 175) and Caspase 8 (Asp 391).

\section{Discussions}

Innovation in the dealing and avoidance of cancer requires the continuous expansion of new cancer protective and curative agents (31). Numbers of constituents isolated from herbs are being used as curing agents against various cancers including breast cancer (32). The worldwide demand of herbal products is increased in past few decades for the management of chronic as well as incurable ailments (33).

Saponins are secondary metabolites usually exist in plants with composite arrangement play a role of defense mechanism and accounted to be active in various dreadful ailments (34). C. fimbriata is a rich source of Saponin and used in this study.

Bioinformatics techniques are gaining importance in the field of new drug development (35). It empowers the researchers to predict pharmacological activities of compounds and allows them to practically monitor a database of millions of different compounds to identify lead molecules. PASSOnline and SwissTargetPredictions are such powerful softwares which could forecast the probabilities of test component to be active against vast number of targets in the body. PASSOnline assigns two probability scores, one for 'Active' and other for 'Inactive'. A compound assigned bigger Pa score and smaller Pi score toward a specific pharmacological activity will be considered for further analysis. SwissTargetPrediction will directly depict the probability to be active score. Structures of Caratubersides $A$ to $G$ were processed through
PASSOnline and SwissTargetPrediction softwares. The predictions related to anticancer activity, which commonly found for all caratubersides, such as antineoplastic for breast cancer, antiangiogenic activity, apoptosis agonist along with caspases 3 and 8 stimulations. Caratuberside A showed the probability of $85.7 \%$ and $71.8 \%$ for stimulating the Caspase 3 and Caspase 8 respectively. While Caratuberside B showed $77.3 \%$ and $69.1 \%$ probability as a stimulator of Caspase 3 and Caspase 8 activity respectively. Rest of the compounds showed probability in decreasing order like Caratuberside $\mathrm{G}>$ Caratuberside $\mathrm{C}>$ Caratuberside $\mathrm{D}>$ Caratuberside $\mathrm{F}>$ Caratuberside E.

Further, we processed these structures for molecular docking by AutoDock Tool and AutoDock Vina. These are freeware which forecasts the affinity of ligands towards the target protein using Lamarckian Genetic algorithm. In molecular docking, ligands are docked with target proteins and the affinities are reported in the form binding free energy. Caratubersides were docked with the identified targets from pharmacological activity predictions i.e. Caspase 3 and Caspase 8. Interestingly lowest binding free energy i.e. -9.4 was found for Caratuberside D with Caspase 3 and -7.9 for Caratuberside E with Caspase 8. Caratubersides $\mathrm{A}$ and $\mathrm{B}$, which showed maximum probability to be active in pharmacological activity prediction, showed binding free energy of -7.9 and -7.9 respectively for Caspase 3 while -7.6 and -6.3 respectively for Caspase 8 (Table 1).

MTT assay is the most preferred and relatively simple method used for cytotoxicity studies. MTT is yellow colored water-soluble dye which is metabolized by the alive cells in purple colored formazan products via $\mathrm{NAD}(\mathrm{P}) \mathrm{H}$-dependent cellular oxidoreductase enzymes (36). We treated MCF-7 cells by different concentrations of saponin rich fraction of $C$. fimbriata till 96 hours and inhibitions in percentage were measured. Graph of percentage inhibition against concentration was designed and IC50 was found to be 3 $\mu \mathrm{g} / \mathrm{ml}$. Earlier Shenai et al., observed the cytotoxicity of ethanolic extract of C. fimbriata leaves in COLO 320 cells, but no reports were found regarding cytotoxicity of saponins from C. fimbriata leaves (1).

Recently, much attention was gain by angiogenesis in cancer biology (37). Oxygen supply and nutrients required for growth and survival of cancer was maintained through sprouting of new blood vessels from the established vasculature and creating a new vascular system within the tumor (38). Moreover, this neovascularization also plays significant role in tumor growth, invasion and metastasis (39). Cancer is considered to be angiogenic dependent disease and most of the studies successfully targeted various regulators of angiogenesis. We exposed shell less chicken embryo cultures at various concentrations $(1.5,3$ and $6 \mu \mathrm{g} / \mathrm{ml})$ of saponin rich fraction and observed considerable $(p<0.05)$ decreases into quantity of angiogenic properties among 0-3 hours as well as 0-6 hours of contact to drug. The test compounds not only prevented the neovascularization but also decreased the number along with length of existing blood vessels. 
Evaluating the occurrence of chromosomal aberrations in peripheral blood is a susceptible cytogenetic test to discover potency of test compounds against mutagenesis and carcinogenesis (40). This kind of assays is generally used in the genotoxicity assessment in human subjects (29). Aberrations in chromosomes were observed, with and without treatment of saponin rich fraction in cultured blood samples. End result revealed non-significant correlation between treated and control groups in chromosomal aberration assay.

Within organisms, pathophysiological conditions can be overcome by various cell death modalities (41). Apoptosis is one such mechanism where genetically synchronized death of the cell acting a vital task in exclusion of infected, injured as well as other unwanted cells from the body (42). Biochemically, the key feature of apoptosis includes activation of cascade of caspase and DNA fragmentation. In human, apoptosis can be instigated by (1) the extrinsic pathway, which can cause caspase 8 stimulation; (2) the intrinsic pathway, started through cellular tension pursued via stimulation of caspase 9; or (3) the granzyme B pathway (43). All such paths meet to a general finishing stage of apoptosis with the aim of proteolytic stimulation of caspases 3. Here saponin rich fraction was assessed for its apoptogenic potential in MCF-7 cells using ELISA. We observed significant stimulation of caspases 3 (OD of 1.35 on $450 \mathrm{~nm}$ ) as well as caspase 8 (OD of 1.68 on $450 \mathrm{~nm}$ ) within test compound treated groups. Caspase 8 is synthesized as inactive zymogen procaspase and gets activated either in extrinsic pathway due to activation of death receptors or it is recruited and activated at the outer membrane of mitochondria in mitochondria mediated apoptosis. It is not clear by which mechanism (intrinsic or extrinsic pathway) saponin rich factor of $C$. fimbriata increases cleaved Caspase 8, but once getting activated Caspase 8 further increases levels of Caspase 3 .

\section{Conclusion}

Results of presented study displayed antiproliferative, cytotoxic, antiangiogenic as well as apoptotic effects of saponin rich fraction of $C$. fimbriata. CAs in normal human blood was nonsignificant, which suggests absence of genotoxicity in normal cells. In silico studies predicted the apoptotic prospective of caratubersides by stimulating caspase 3 and caspase 8 . The results obtained for saponin rich fraction of $C$. fimbriata is in accordance with this prediction and showed significantly increased levels of caspases 3 along with caspase 8 in breast cancer MCF-7 cell line.

\section{Conflict of interest}

The authors declared no conflict of interest.

\section{References}

1. Shenai A, Devaraj E, Roy A. Cytotoxic Effect of Caralluma fimbriata Against Human Colon Cancer Cells. Pharmacogn J 2017; 9(2): 204-207.
2. Odendaal AY, Deshmukh NS, Marx TK, Schauss AG, Endres JR, Clewell AE. Safety assessment of a hydroethanolic extract of Caralluma fimbriata. Int $\mathbf{J}$ Toxicol 2013; 32(5): 385-394.

3. Padwal AD, Varpe SN, Waman MB. Phytochemical and Nutritional Analysis of Caralluma Fimbriata L. Int J Res Biosci Agric Technol 2016; 4(1): 193-5

4. Naingade SS, Jadhav AS, Surve SB. Caralluma fimbriata: AN OVERVIEW. Int J Pharm Bio Sci 2013; 3(1): 281-286.

5. Gujjala S, Putakala M, Nukala S, Bangeppagari M, Ramaswamy R, Desireddy S. Renoprotective effect of Caralluma fimbriata against high-fat diet-induced oxidative stress in Wistar rats. J Food Drug Anal 2016; 24(3): 586-593.

6. Latha S, Rajaram K, Suresh KP. Hepatoprotective and Antidiabetic Effect of Methanol Extract of Caralluma fimbriata in streptatozocin induced diabetic albino rats. Int J Pharm Pharm Sci 2014; 6(1): 665-668.

7. Kamalakkannan S, Rajendran R, Venkatesh RV, Clayton P, Akbarsha MA. Antiobesogenic and Antiatherosclerotic Properties of Caralluma fimbriata Extract. J Nutr Metab 2010; 2010: 285301.

8. Priya D, Rajaram K, Suresh KP. Phytochemical studies and GC-MS analysis of Caralluma fimbriata wall. Int. J. Pharm. Res. Develop 2011; 3(10): 105-110.

9. Lakshmi T, Rajendran R, Raghavan V, Silvester A. HPTLC fingerprinting method to distinguish total extract of Caralluma fimbriata from the modified extracts of Caralluma fimbriata. Biosci Biotech Res Asia 2014; 11(2): 785-789.

10. Sudhakara G, Mallaiah P, Sreenivasulu N, Rao SB, Rajendran R, Saralakumari D. Beneficial effects of hydro-alcoholic extract of Caralluma fimbriata against high-fat diet-induced insulin resistance and oxidative stress in Wistar male rats. J Physiol Biochem 2014; 70(2): 311-320.

11. Devi SG, Dhamotharan R. Caralluma fimbriata- An Important Medicinal Plant: A Review of Its Traditional Uses, Phytochemistry and Pharmacological Properties. Int J Pharmtech Res 2016; 9: 223-230.

12. Qayyum N, Rani H. Mir KB, Khan AQ. Caralluma Pharmacological Attributes. J Food Nutr Popul Health 2018; 2: 13-15.

13. Zari, T. A.; Al-Thebaiti, M. A. Effects of Caralluma russeliana stem extract on some physiological parameters in streptozotocininduced diabetic male rats. Diabetes Metab Syndr Obes 2018; 11: 619-631.

14. Al-Faifi ZIA, Masrahi YS, Aly MS, Al-Turki TA. In vitro Anticancer Activity of Caralluma acutangula (Decne.) N.E.Br. Extract. Int J Phar Sci Rev Res 2016; 38: 59-63.

15. Rajendran R, Ambikar DB, Khandare RA, Sannapuri VD, Vyawahare NS, Clayton PR. Nootropic Activity of Caralluma fimbriata Extract in Mice. Food Nutr Sci 2014; 5(2): 147-152.

16. EFSA Panel on Dietetic Products, Nutrition and Allergies (NDA); Scientific Opinion on thesubstantiation of a health claim related to ethanolwater extract of Caralluma fimbriata (Slimaluma ${ }^{\circledR}$ ) and helps to control hunger/appetite pursuant to 
Article 13(5) of Regulation (EC) No 1924/2006. EFSA Journal 2010; 8: 1606.

17. Dutt HC, Singh S, Avula B, Khan IA, Bedi YS. Pharmacological Review of Caralluma R.Br. with Special Reference to Appetite Suppression and AntiObesity. J Med Food 2011; 15(2): 108-119.

18. Desai TH, Joshi SV. Anticancer activity of saponin isolated from Albizia lebbeck using various in vitro models. J Ethnopharmacol 2019; 231: 494-502.

19. Xu XH, Li T, Fong CMV, Chen X, Chen XJ, Wang YT, et al. Saponins from chinese medicines as anticancer agents. Molecules 2016; 21(10): E1326.

20. Pal BC, Achari B, Yoshikawa K, Arihara S. Saponins from Albizia lebbeck. Phytochemistry 1995; 38(5): 1287-1291.

21. Mallick SS, Vidya VD. Detection and Estimation of alpha-Amyrin, beta-Sitosterol, Lupeol, and nTriacontane in Two Medicinal Plants by High Performance Thin Layer Chromatography. Adv Chemistry 2014; 2014: 1-7.

22. Lagunin A, Zakharov A, Filimonov D, Poroikov V. QSAR modelling of rat acute toxicity on the basis of PASS prediction. Mol Inform 2011; 30: 241-520.

23. Poroikov V, Filimonov D, Borodina YV, Lagunin A. A: Robustness of biological activity spectra predicting by computer program PASS for noncongeneric sets of chemical compounds. J Chem Inf Comput Sci 2000; 40: $1349-1355$.

24. Poroikov VV, Filimonov DA, Ihlenfeldt WD, Gloriozova TA, Lagunin AA, Borodina YV, Stepanchikova AV, Nicklaus MC. PASS biological activity spectrum predictions in the enhanced open NCI database browser. J Chem Inf Comput Sci 2003; 43: $228-236$.

25. Trott O, Olson AJ. AutoDock Vina: improving the speed and accuracy of docking with a new scoring function, efficient optimization, and multithreading. J Comput Chem 2010; 31: 455-461.

26. Laskowski RA, Swindells MB. LigPlot+: multiple ligand-protein interaction diagrams for drug discovery. J Chem Inf Model 2011; 51: 2778-2786.

27. Plumbs JA. Cell sensitivity assay: The MTT assay. Methods in molecular medicine, cytotoxic drug resistance mechanisms, Humana Press, New Jersey. Vol 28.

28. Brahma J, Dhumal K. Evaluation of Anti-angiogenic properties of Tridax procumbens leaves extract using shell less chick embryo culture. Int J Bioassays 2014; 3(02): 1765-1767.

29. Alsatari ES, Mohammad A, Khabour OF, Alzoubi KH, Sadiq MF. Assessment of DNA damage using chromosomal aberrations assay in lymphocytes of waterpipe smokers. Int J Occup Med Environ Health 2012; 25(3): 218-224.

30. Xu JX, Song HP, Bu QX, Feng DP, Xu XF, Sun OR, $\mathrm{Li}$ XL. Isoflavone Attenuates the Caspase-1 and
Caspase-3 Level in Cell Model of Parkinsonism. Behav Neurol 2015; 2015: 725897.

31. Lacaille-Dubois MA. Bioactive saponins with cancer related and immunomodulatory activity: Recent developments. Studies in Natural Products Chemistry 32(PART L), 2005, pp. 209-246.

32. Cheng L, Xia TS, Wang YF, Zhou W, Liang XQ, Xue $J$ Q, et al. The apoptotic effect of D Rhamnose $\beta$ hederin, a novel oleanane-type triterpenoid saponin on breast cancer cells. PLoS One 2014; 9(3): e90848.

33. Kanchana A, Balakrishnan M. Anti-cancer effect of saponins isolated from Solanum trilobatum leaf extract and induction of apoptosis in human larynx cancer cell lines. Int J Pharm Pharm Sci 2011; 3(4): 356-364.

34. Gao JL, Lv GY, He BC, Zhang BQ, Zhang H, Wang $\mathrm{N}$, et al. Ginseng saponin metabolite 20(S)protopanaxadiol inhibits tumor growth by targeting multiple cancer signaling pathways. Oncol Rep 2013; 30(1): 292-298.

35. Shenai A, Varkey SP, Shantaram M. In Silico Docking of Polyphenolic Compounds against Caspase 3-HeLa Cell Line Protein. Int J Drug Dev \& Res 2017; 9(3): 28-32.

36. Bahuguna A, Khan I, Bajpai VK, Kang SC. MTT assay to evaluate the cytotoxic potential of a drug. Bangladesh J Pharmacol 2017; 12(2): 115-118.

37. Keshavarz M, Bidmeshkipour A, Mostafaie A, Mansouri K, Mohammadi-Motlagh H. Anti-Tumor Activity of Salvia officinalis is Due to Its AntiAngiogenic, Anti-Migratory and Anti-Proliferative Effects. Cell J 2011; 12(4): 477-482.

38. Tantiado RG, Tan VP. Evaluation of the angiosuppresive activity of Tinospora rumphii Boerl. stem extract using the chorioallantoic membrane assay in Anas platyrhynchos embryos. Intl $\mathrm{J}$ Biosci and Biotechnol 2012; 4(2): 93-102.

39. Kota K, Sharma S, Ragavendhra P. Study of antiangiogenic activity of "aqueous extract of Nigella sativa seeds" in chick chorioallantoic membrane (CAM) model. Int J Adv Med 2018; 5(4): 895.

40. Bonassi S, Abbondandolo A, Camurri L., Dal PL, De FM, Degrassi F, et al. Are Chromosome Aberrations in Circulating Lymphocytes Predictive of Future Cancer Onset in Humans? Preliminary Results of an Italian Cohort Study. Cancer Genet Cytogenet 1995; 79(2): 133-135.

41. Olsson M, Zhivotovsky B. Caspases and cancer. Cell Death Differ 2011; 18(9): 1441-1449.

42. Devarajan E, Sahin AA, Chen JS, Krishnamurthy RR, Aggarwal N, Brun A, et al. Down-regulation of caspase 3 in breast cancer: a possible mechanism for chemoresistance. Oncogene 2002; 21(57): 8843-8851.

43. Ghavami S, Hashemi M, Ande SR, Yeganeh B, Xiao W, Eshraghi M, et al. Apoptosis and cancer: mutations within caspase genes. J Med Genet 2009; 46: 497510 . 